\title{
Melatonin alleviates lipopolysaccharide-induced myocardial injury by inhibiting inflammation and pyroptosis in cardiomyocytes
}

\author{
Ze-Da-Zhong Su${ }^{1,2 \#}$, Xue-Biao Wei ${ }^{2,3 \#}$, Yan-Bin $\mathrm{Fu}^{1,2 \#}$, Jia $\mathrm{Xu}^{4}$, Zhong-Hua Wang ${ }^{3}$, Yu Wang ${ }^{2}$, \\ Jian-Feng $\mathrm{CaO}^{2}$, Jie-Leng Huang ${ }^{2}$, Dan-Qing $\mathrm{Yu}^{1,2}$
}

${ }^{1}$ Guangdong Provincial People's Hospital, Guangdong Academy of Medical Sciences, School of Medicine, South China University of Technology, Guangzhou, China; ${ }^{2}$ Department of Cardiology, Guangdong Cardiovascular Institute, Guangdong Provincial Key Laboratory of Coronary Heart Disease Prevention, Guangdong Provincial People’s Hospital, Guangdong Academy of Medical Sciences, Guangzhou, China; ${ }^{3}$ Guangdong Provincial Geriatrics Institute, Guangdong Provincial People's Hospital, Guangdong Academy of Medical Sciences, Guangzhou, China; ${ }^{4}$ Department of Emergency, The First Affiliated Hospital of Sun Yat-sen University, Guangzhou, China

Contributions: (I) Conception and design: JL Huang, DQ Yu, XB Wei, ZDZ Su; (II) Administrative support: YB Fu, J Xu, ZH Wang; (III) Provision of study materials or patients: Y Wang, JF Cao; (IV) Collection and assembly of data: ZDZ Su, XB Wei, YB Fu; (V) Data analysis and interpretation: ZDZ Su, XB Wei, YB Fu; (VI) Manuscript writing: All authors; (VII) Final approval of manuscript: All authors.

"These authors are considered as co-first authors.

Correspondence to: Dan-Qing Yu, MD, PhD. Guangdong Provincial People's Hospital, Guangdong Academy of Medical Sciences, School of Medicine, South China University of Technology, Guangzhou, China; Department of Cardiology, Guangdong Cardiovascular Institute, Guangdong Provincial Key Laboratory of Coronary Heart Disease Prevention, Guangdong Provincial People's Hospital, Guangdong Academy of Medical Sciences, Guangzhou 510080, China. Email: gdydq100@126.com; Jie-Leng Huang, MD. Department of Cardiology, Guangdong Cardiovascular Institute, Guangdong Provincial Key Laboratory of Coronary Heart Disease Prevention, Guangdong Provincial People's Hospital, Guangdong Academy of Medical Sciences, Guangzhou 510080, China. Email: jieleng@126.com.

Background: Melatonin (MT) has been shown to protect against various cardiovascular diseases. However, the effect of MT on lipopolysaccharide (LPS)-induced myocardial injury is poorly understood. This study aims to evaluate the effects of MT on LPS-induced myocardial injury in vitro.

Methods: H9C2 cells were divided into a control group, MT group, LPS group, and MT + LPS group. The control group was treated with sterile saline solution, the LPS group received $8 \mu \mathrm{g} / \mathrm{mL}$ LPS for $24 \mathrm{~h}$, MT + LPS cells were pretreated with $200 \mu \mathrm{mol} / \mathrm{L} \mathrm{MT}$ for $2 \mathrm{~h}$ then with $8 \mu \mathrm{g} / \mathrm{mL}$ LPS for $24 \mathrm{~h}$, and the MT group received only $200 \mu \mathrm{mol} / \mathrm{L} \mathrm{MT}$ for $2 \mathrm{~h}$. The CCK-8 assay and lactate dehydrogenase (LDH) activity assay were used to analyze cell viability and LDH release, respectively. Intracellular reactive oxygen species (ROS) and the rate of pyroptosis were measured using the fluorescent probe dichloro-dihydro-fluorescein diacetate (DCFH-DA) and propidium iodide (PI) staining, respectively. The cell supernatants were used to measure the levels of inflammatory cytokines, including IL-6, TNF- $\alpha$, and IL-1 $\beta$ by enzyme-linked

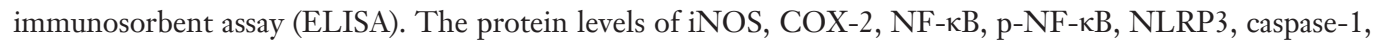
and GSDMD were detected by western blot.

Results: MT pretreatment significantly improved LPS-induced myocardial injury by inhibiting

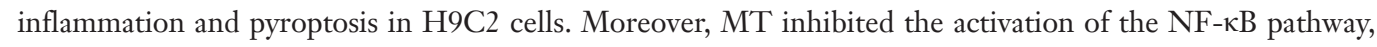
and reduced the expression of inflammation-related proteins (iNOS and COX-2), and pyroptosis-related proteins (NLRP3, caspase-1, and GSDMD).

Conclusions: Our data suggests that MT can alleviate LPS-induced myocardial injury, providing novel insights into the treatment of sepsis-induced myocardial dysfunction.

Keywords: Melatonin (MT); lipopolysaccharide (LPS); myocardial injury; inflammation; pyroptosis

Submitted Dec 02, 2020. Accepted for publication Feb 03, 2021.

doi: 10.21037/atm-20-8196

View this article at: http://dx.doi.org/10.21037/atm-20-8196 


\section{Introduction}

Sepsis is a complex life-threatening organ dysfunction syndrome caused by a dysregulated host response to infection that leads to an uncontrolled immune response (1). Cardiac dysfunction is prevalent in patients with sepsis $(2,3)$, who have a poor prognosis $(4,5)$. Therefore, the survival of septic patients may be associated with improving cardiac function.

Myocardial injury is commonly observed in patients with sepsis, which is mainly induced by lipopolysaccharide (LPS), a bacterial endotoxin found in the cell wall of Gramnegative bacteria (6-8). In cardiomyocytes, LPS activates nuclear factor- $\kappa \mathrm{B}(\mathrm{NF}-\kappa \mathrm{B})$ which then increases the expression of inducible nitric oxide synthase (iNOS) and cyclooxygenase-2 (COX-2), speeding up the synthesis and release of inflammatory cytokines $(6,9)$. The overexpression and release of proinflammatory cytokines induced by LPS, such as interleukin-6 (IL-6) and tumor necrosis factor- $\alpha$ (TNF- $\alpha$ ), have been associated with cardiac dysfunction, including myocardial depression (10). In addition, Sepsis is closely associated with pyroptosis, which is a novel type of programmed cell death (11). ROS-dependent pyroptosis has also been recognized as an important pathway mediating LPS-induced myocardial injury (12). Pathogen-associated molecular patterns (PAMPs), such as LPS, could induce reactive oxygen species (ROS) production, which can initiate NLR family pyrin domain containing 3 (NLRP3) inflammasome activation then activating caspase-1, subsequently promoting pyroptosis and the secretion of IL-1 $\beta(13-17)$. ROS inhibitors have been reported to downregulate NLRP3 mRNA expression, indicating that NLRP3 gene expression is partly modulated by ROS generation $(18,19)$. Accordingly, inhibiting the inflammation and ROS-dependent pyroptosis in cardiomyocytes may be a promising treatment for sepsis-induced myocardial injury.

Melatonin (MT) is a vital neuroendocrine hormone synthesized by the pineal gland (20). Although the primary functions of MT are associated with sleep cycle regulation (21), it recently has shown strong effects of anti-inflammation, anti-oxidation, alleviating adipose and endothelial pyroptosis (22-25). Several studies have shown that MT could protect against myocardial injury (26-30) via regulating apoptosis, autophagy, improving mitochondrial function. However, the underlying mechanisms that MT improves LPS-induced myocardial injury remain unclear. In particular, no study has found that melatonin could alleviate LPS-induced myocardial injury by inhibiting inflammation and pyroptosis, simultaneously.
In this study, we hypothesize that MT attenuates LPSinduced myocardial injury by inhibiting NF- $\kappa \mathrm{B} / \mathrm{iNOS} /$ COX-2-induced inflammation and ROS-dependent NLRP3-mediated pyroptosis in H9C2 cardiomyocytes. In addition, we present the following article in accordance with the MDAR checklist (available at http://dx.doi. org/10.21037/atm-20-8196).

\section{Methods}

\section{Cell culture}

The H9C2 cells (Cell Bank of the Chinese Academy of Sciences, Shanghai, China) were cultured in DMEM (GIBCO, USA) supplemented with 10\% fetal bovine serum (FBS) and $1 \%$ penicillin/streptomycin in a humidified atmosphere containing $5 \% \mathrm{CO}_{2} / 95 \%$ air at $37{ }^{\circ} \mathrm{C}$. Moreover, $0.05 \%$ trypsin was used to digest cells, and cell medium was replaced every 3 days. H9C2 cells were treated with different stimuli in 6-well plates or 96-well plates.

\section{Treatment of cells}

LPS (Sigma, USA) and MT (Sigma, USA) were dissolved in sterile deionized water to obtain a final concentration of $8 \mu \mathrm{g} / \mathrm{mL}$ and $200 \mu \mathrm{mol} / \mathrm{L}$, respectively. The control group was treated with sterile saline solution, and the LPS group received $8 \mu \mathrm{g} / \mathrm{mL}$ LPS for $24 \mathrm{~h}$. Cells in the MT + LPS group were pretreated with $200 \mu \mathrm{mol} / \mathrm{L} \mathrm{MT}$ for $2 \mathrm{~h}$, then $8 \mu \mathrm{g} / \mathrm{mL}$ LPS for $24 \mathrm{~h}$. The MT group received only $200 \mu \mathrm{mol} / \mathrm{L}$ MT for $2 \mathrm{~h}$. After incubation, cells were collected, and lactate dehydrogenase (LDH) levels, ROS levels, cell viability, and protein expression were analyzed. The levels of superoxide dismutase (SOD), malondialdehyde (MDA), IL-6, $\mathrm{TNF}-\alpha$, and IL- $1 \beta$ in culture medium were estimated.

\section{Cell viability}

According to the manufacturer's instructions, CCK-8 assay kit (Bestbio, Shanghai, China) was used to determine the cell viability in 96-well plates. Twenty four hours after cell treatment, $10 \mu \mathrm{L}$ of sterile CCK- 8 was added to each well and incubated in the dark for $2 \mathrm{~h}$ at $37^{\circ} \mathrm{C}$. The absorbance at $450 \mathrm{~nm}$ was determined using a microplate reader.

\section{LDH activity assay}

LDH activity was detected to estimate cell injury by a LDH 
assay kit (BeastBio, Shanghai, China) according to the manufacturer's instruction.

\section{Enzyme-linked immunosorbent assay (ELISA)}

Commercial ELISA kits (Jianglai, Shanghai, China) were used to measure the concentrations of inflammatory cytokines, including TNF- $\alpha$, IL- 6 , and IL- $1 \beta$, and oxidative stress biomarkers such as SOD and MDA.

\section{Measurement of ROS generation}

The levels of intracellular ROS were measured with the fluorescent probe dichloro-dihydro-fluorescein diacetate (DCFH-DA; Sigma, USA). After treatment with stimuli, cells were incubated with DCFH-DA $(50 \mu \mathrm{M})$ at $37^{\circ} \mathrm{C}$ for $30 \mathrm{~min}$. Then, PBS was used to wash the cells twice. The average fluorescence intensity was analyzed using an image analysis system.

\section{Western blot}

After treatment with the stimuli, radioimmunoprecipitation assay (RIPA) buffer (Beyotime, China), containing protease inhibitor cocktail (Beyotime, China) including phenylmethylsulfonyl fluoride (PMSF; Beyotime, China), was used to lyse the cells, and all homogenates were centrifuged for $15 \mathrm{~min}$ at $12,000 \mathrm{rpm}$ at $4{ }^{\circ} \mathrm{C}$. $10 \%$ SDSPAGE gels were used to load equal amounts of denatured protein, which was then transferred onto polyvinylidene difluoride (PVDF) membranes. 5\% nonfat milk was applied to block membranes at room temperature for $1 \mathrm{~h}$. The membranes were then incubated overnight with primary antibodies against iNOS (1:1,000, NOVUS, USA), COX-2 (1:500, Abcam, USA), NF-кB (1:1,000, Abcam, USA), p-NF-кB (1:1,000, Abcam, USA), NLRP3 (1:1,000, NOVUS, USA), caspase-1 (1:1,000, NOVUS, USA), GSDMD (1:1000, Abcam, USA), and GAPDH $(1: 10,000$, Abcam, USA). A fluorescent secondary antibody (1:2,500, CST, USA) was subsequently used to incubate membranes for $2 \mathrm{~h}$ at $4{ }^{\circ} \mathrm{C}$. Then, TBST was used to wash the membranes 3 times, each time for 5 minutes. The quantification of protein expression was normalized to GAPDH.

\section{Propidium iodide (PI) staining}

After stimulation, the cells were collected and washed 3 times with $1 \times$ PBS. Then, $1 \times$ assay buffer was used to wash the cells. $5 \mu \mathrm{M}$ PI were then mixed with $1 \times$ assay buffer and stained per well at $37^{\circ} \mathrm{C}$ for $15 \mathrm{~min}$. Finally, DAPI (Sigma, USA) were used to stain cells for $5 \mathrm{~min}$. A fluorescence microscope was used to acquire the images of the cells immediately after staining. The the average fluorescence intensity and the percentage of positive cells were assessed with Image J.

\section{Statistical analysis}

All values are expressed as the mean $\pm \mathrm{SD}$. The statistical analyses were done by GraphPad Prism version 8.0 (GraphPad Software, USA). One-way ANOVA or twoway ANOVA followed by Tukey's test were performed to analyze the differences among experimental groups. $\mathrm{P}$ values $<0.05$ were considered to be statistically significant.

\section{Results}

\section{The effect of LPS on cell viability and LDH release in H9C2 cells}

To confirm the effects of LPS on cellular activity and LDH release, cardiomyocytes were exposed to different concentrations of LPS. The viability of H9C2 cells was negatively correlated with LPS concentration. In response to $8 \mu \mathrm{g} / \mathrm{mL}$ LPS, cell viability was significantly lower compared to the control group (Figure 1A). In addition, $\mathrm{LDH}$ release increased in a concentration-dependent manner (Figure $1 B$ ), and the highest $\mathrm{LDH}$ release was observed after treating cells with $8 \mu \mathrm{g} / \mathrm{mL}$ LPS.

\section{The effect of LPS on inflammatory cytokines in cell supernatants and oxidative stress biomarkers in $\mathrm{H} 9 \mathrm{C} 2$ cells}

After treating cells with different concentrations of LPS, the supernatants were collected to identify the levels of inflammatory cytokines. IL- 6 and TNF- $\alpha$ levels were positively correlated with LPS concentration (Figure 1C,D). Furthermore, the levels of ROS increased in a dose-dependent manner (Figure 1E), the activity of SOD decreased (Figure $1 F$ ), and the levels of MDA (Figure 1G) and IL- $\beta$ (Figure $1 H$ ) increased.

\section{The effect of MT on cell viability and $L D H$ release in LPS-treated H9C2 cells}

When cells were treated with MT (50-200 $\mu \mathrm{mol} / \mathrm{L})$ for 


\section{A}

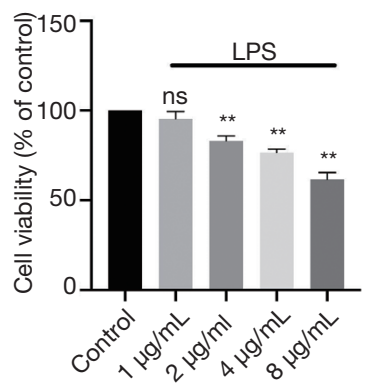

C

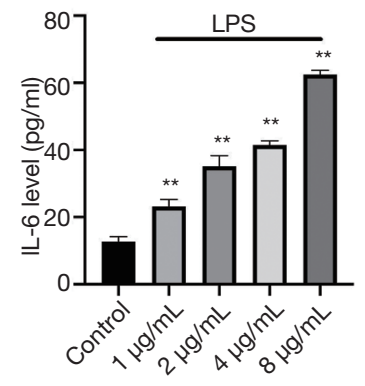

B
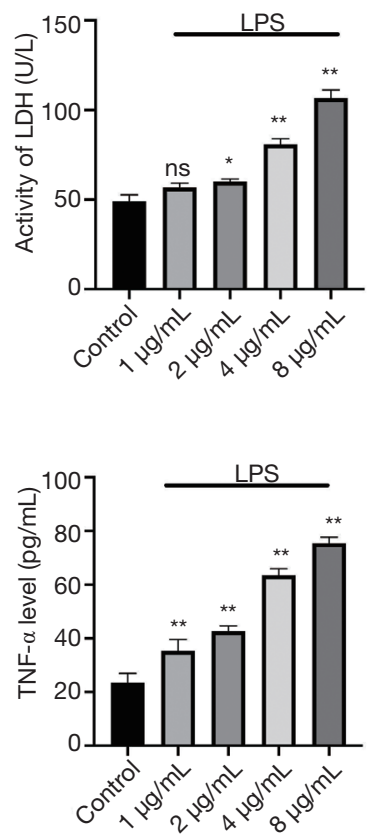

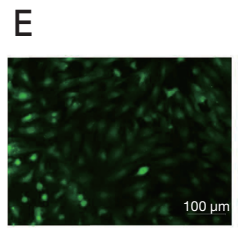

Control

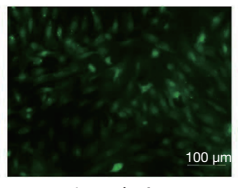

$1 \mu \mathrm{g} / \mathrm{mL}$

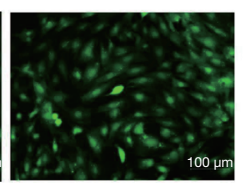

$2 \mu \mathrm{g} / \mathrm{mL}$

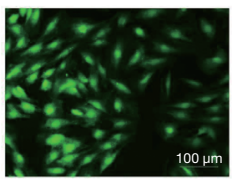

$4 \mu \mathrm{g} / \mathrm{mL}$

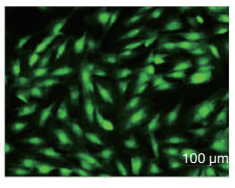

$8 \mu \mathrm{g} / \mathrm{mL}$

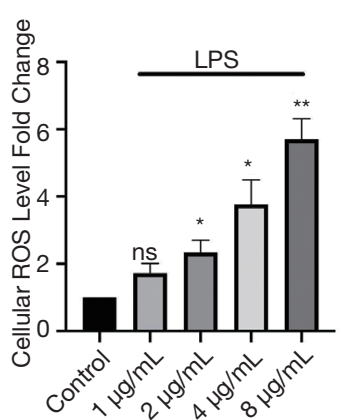

G

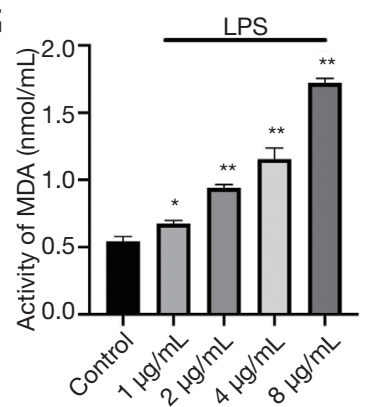

$\mathrm{H}$

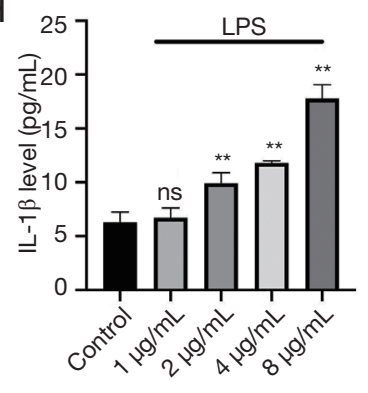

Figure 1 Dose-dependent effects of lipopolysaccharide (LPS) on H9C2 cells. (A) Cell viability was measured using the CCK-8 assay. (B) Lactate dehydrogenase (LDH) release was detected by LDH activity assays. (C,D) The levels of inflammatory cytokines, including IL-6 and TNF- $\alpha$, were detected by ELISA. (E) The levels of intracellular reactive oxygen species (ROS) were detected by fluorescent probe dichlorodihydro-fluorescein diacetate (DCFH-DA) (scale bar: $100 \mu \mathrm{m}$. 100x magnification). (F) The activity of superoxide dismutase (SOD), (G) the content of malondialdehyde (MDA), and (H) the levels of IL- $\beta$ were detected by ELISA. Data are shown as mean \pm standard deviation (SD) $(\mathrm{n}=6)$. *, $\mathrm{P}<0.05$ and ${ }^{* *}, \mathrm{P}<0.01$ compared to control group; $\mathrm{ns}, \mathrm{P}>0.05$ compared to control group. 

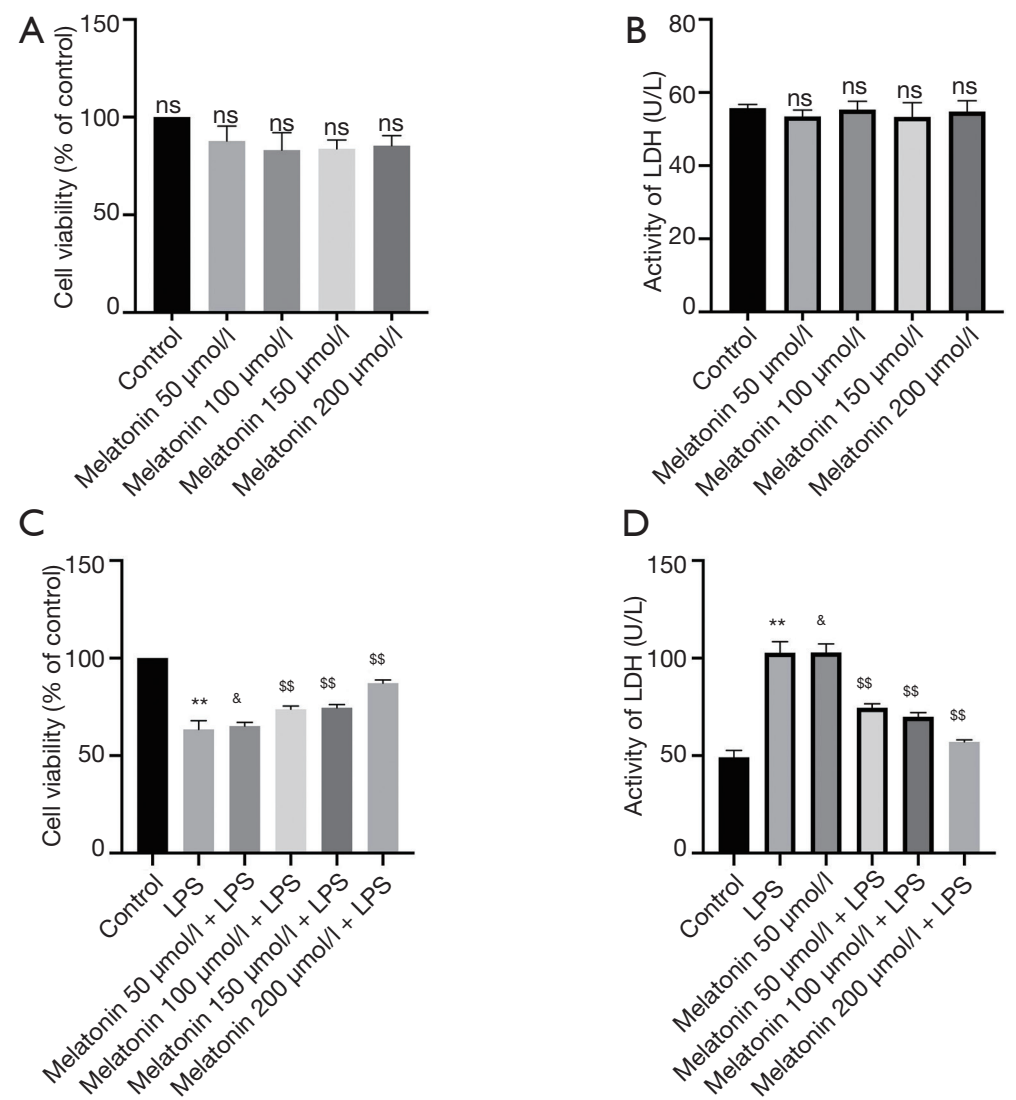

Figure 2 The effect of melatonin (MT) on cell viability and lactate dehydrogenase (LDH) release in lipopolysaccharide (LPS)-treated H9C2 cells. (A) The toxicity of MT on cells was measured using the CCK-8 assay and (B) the activity of LDH. (C) The effects of MT on LPS-induced H9C2 cell injury were detected using the CCK-8 assay and (D) LDH release. Data are shown as mean \pm standard deviation (SD) ( $\mathrm{n}=6) .{ }^{* *}, \mathrm{P}<0.01$ compared to control group; ${ }^{\$ \$}, \mathrm{P}<0.01$ compared to LPS group; ns, $\mathrm{P}>0.05$ compared to control group; ${ }^{\&}, \mathrm{P}>0.05$ compared to LPS group.

$24 \mathrm{~h}$, cell viability and LDH release did not significantly change (Figure $2 A, B$ ). Subsequently, we evaluated the effect of MT on LPS-induced cardiomyocyte injury. Pretreatment with MT for $2 \mathrm{~h}$ significantly protected cells from LPS-induced injury in a concentration-dependent manner. Cell viability gradually recovered, and LDH release decreased, compared to the LPS group. Statistically, a significant inhibitory effect of MT on cytotoxicity commenced at $200 \mu \mathrm{mol} / \mathrm{L}$ (Figure 2C,D).

\section{The effect of MT on inflammatory cytokines in cell supernatants}

Inflammatory responses and cytokine release play a crucial role in LPS-induced cell injury. After LPS treatment, the expression of inflammatory cytokines (IL-6, TNF- $\alpha$ ) in cell supernatants dramatically increased. In contrast, MT (200 $\mu \mathrm{mol} / \mathrm{L})$ pretreatment down-regulated IL-6 and TNF- $\alpha$ levels (Figure $3 A, B$ ).

\section{The effect of MT on the expression of iNOS, COX-2, and} the activation of the NF- $\mathrm{KB}$ pathway in LPS-treated H9C2 cells

To investigate the possible anti-inflammatory mechanisms of MT on LPS-induced myocardial injury, we explored the protein expression of iNOS and COX-2, and the activation of NF- $\mathrm{KB}$ in cardiomyocytes. After exposed to LPS, the levels of iNOS, COX-2, and $\mathrm{p}-\mathrm{NF}-\mathrm{\kappa B}$ were upregulated. This effect was reversed after adding MT (Figure $4 A, B, C, D, E)$. 

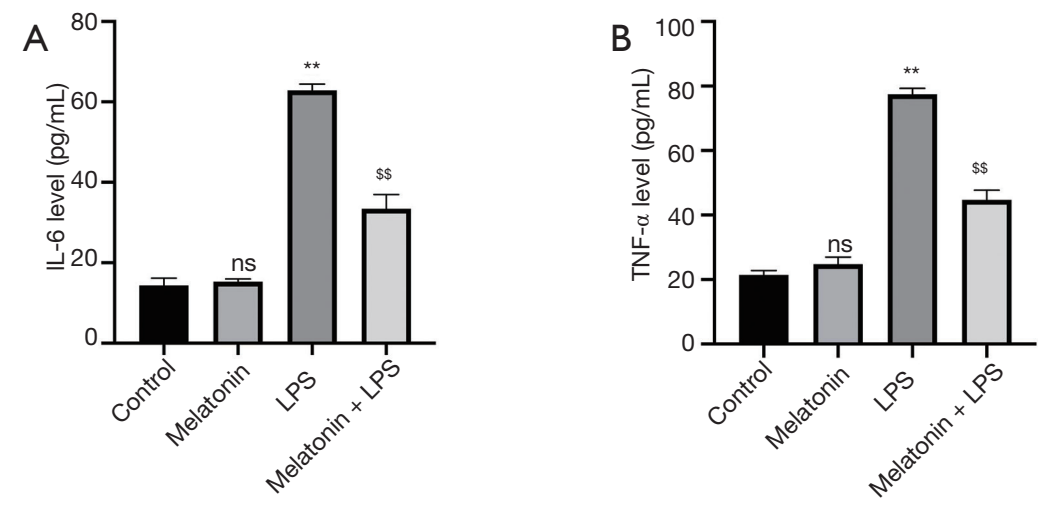

Figure 3 The effect of melatonin on interleukin-6 (IL-6) and tumor necrosis factor- $\alpha$ (TNF- $\alpha$ ) in lipopolysaccharide (LPS)-treated H9C2 cells. The levels of (A) IL-6 and (B) TNF- $\alpha$ in cell supernatants were determined by ELISA. Values were shown as mean \pm standard deviation (SD) ( $\mathrm{n}=6)$. **, $\mathrm{P}<0.01$ compared to control group; ${ }^{\$ \$}, \mathrm{P}<0.01$ compared to LPS group; ns, $\mathrm{P}>0.05$ compared to control group.
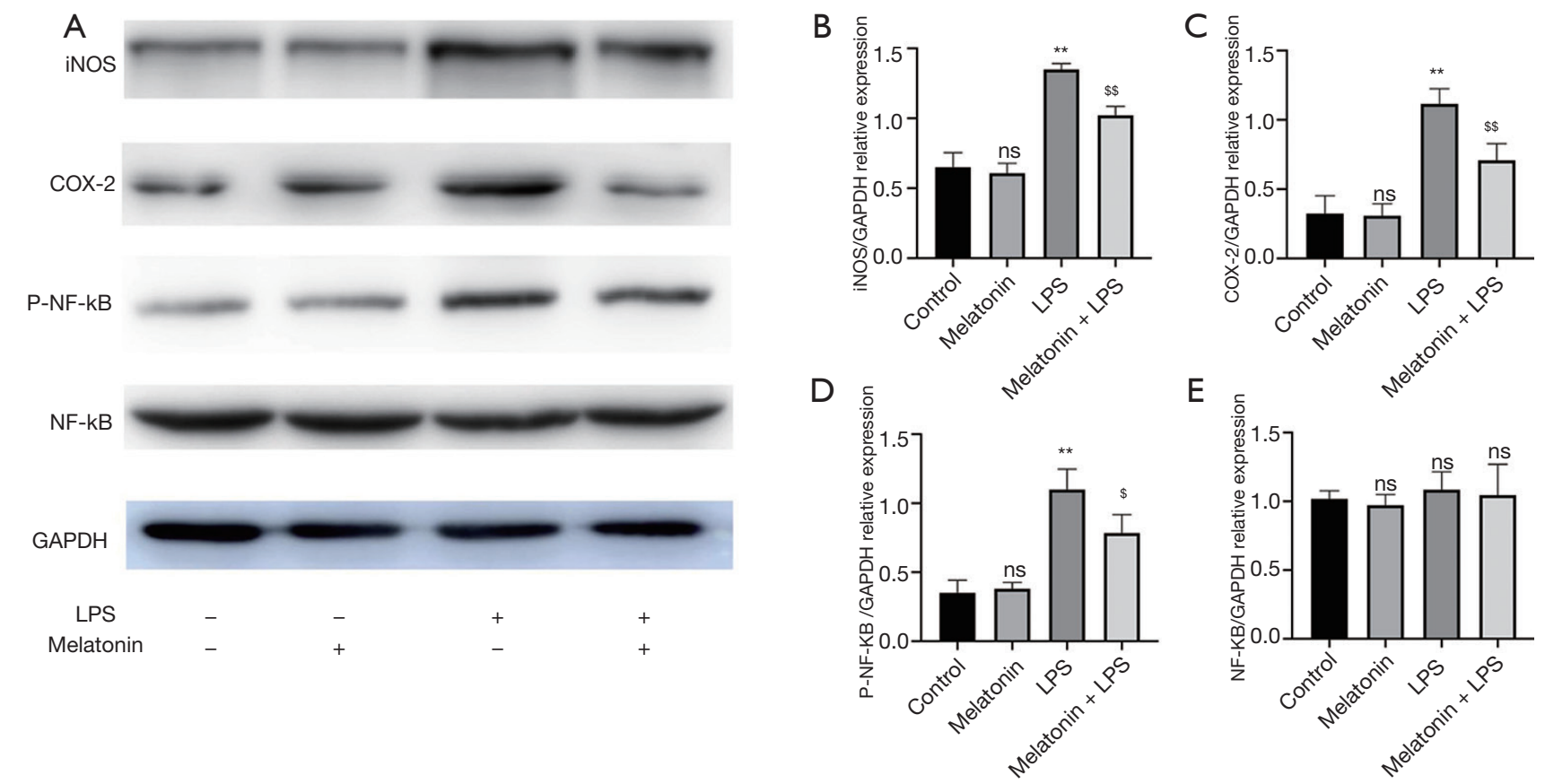

Figure 4 The effect of melatonin on the expression of inducible nitric oxide synthase (iNOS), cyclooxygenase-2 (COX-2), nuclear factor$\kappa \mathrm{B}(\mathrm{NF}-\kappa \mathrm{B})$ and $\mathrm{p}-\mathrm{NF}-\kappa \mathrm{B}$ in lipopolysaccharide (LPS)-treated H9C2 cells. A “+” symbol indicates presence and a “-” symbol indicates the absence of the relevant treatment. (A) iNOS, COX-2, NF- $\kappa$ B and p-NF- $\kappa$ B protein expression in H9C2 was analyzed by western blot. The relative protein expression levels of (B) iNOS/GAPDH, (C) COX-2/GAPDH, (D) p-p65/GAPDH, and (E) p65/GAPDH in H9C2 cells were analyzed. Data are shown as mean \pm standard deviation $(\mathrm{SD})(\mathrm{n}=3)$. ${ }^{* *}, \mathrm{P}<0.01$ compared to control group; ${ }^{\mathrm{s}}, \mathrm{P}<0.05$ compared to LPS group; ${ }^{\$}, \mathrm{P}<0.01$ compared to LPS group; ns, $\mathrm{P}>0.05$ compared to control group.

\section{The effect of MT on ROS-dependent pyroptosis in LPS- treated $\mathrm{H} 9 \mathrm{C} 2$ cells}

We then investigated the role of ROS in LPS-induced myocardial injury. LPS treatment increased intracellular
ROS and MDA levels, and reduced SOD activity in H9C2 cells. Nevertheless, MT pretreatment effectively reduced the production of MDA and ROS, and also improved the activity of SOD (Figure $5 A, B, C$ ). Furthermore, the number 


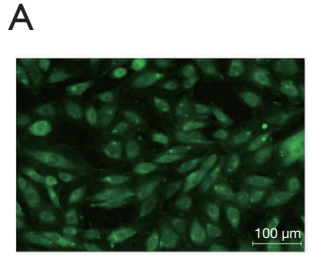

Control

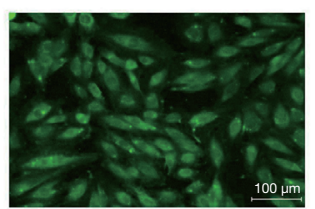

Melatonin

B

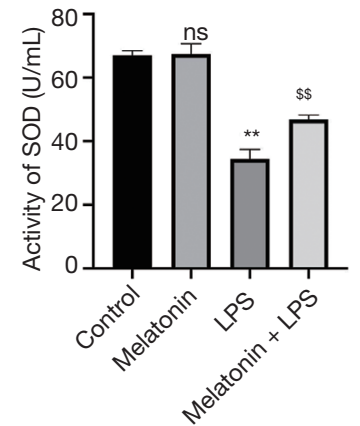

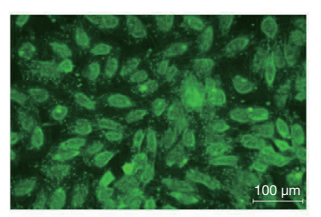

LPS

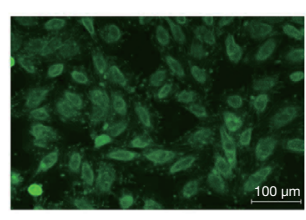

Melatonin + LPS

C

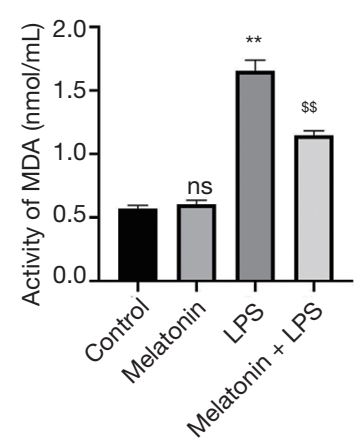

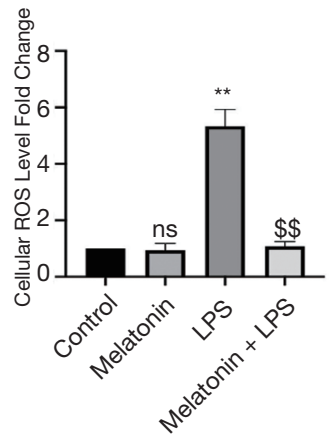

Figure 5 The effect of melatonin on the levels of intracellular reactive oxygen species (ROS), malondialdehyde (MDA), and the activity of superoxide dismutase (SOD) in lipopolysaccharide (LPS)-treated H9C2 cells. (A) ROS levels were measured by DCFH-DA (scale bar: $100 \mu \mathrm{m} .200 \times$ magnification). (B) SOD activity and (C) MDA levels were measured using ELISA. Data are shown as mean \pm standard deviation $(\mathrm{SD})(\mathrm{n}=6){ }^{* *}, \mathrm{P}<0.01$ compared to control group; ${ }^{\text {s\$ }}, \mathrm{P}<0.01$ compared to LPS group; ns, $\mathrm{P}>0.05$ compared to control group.

of pyroptotic cardiomyocytes dramatically increased with the production of increased ROS stimulated by LPS. By contrast, MT alleviated cardiomyocyte pyroptosis by reducing ROS (Figures $5 \mathrm{~A}$ and 6 ). The results indicated the MT improved cellular activity by inhibiting ROSdependent pyroptosis.

\section{The effect of MT on the expression of NLRP3, caspase-1, and GSDMD in LPS-treated H9C2 cells}

The above results demonstrated that MT alleviated H9C2 cell pyroptosis by reducing the production of intracellular ROS. Thus, in the next experiment, pyroptosis proteins including NLRP3, caspase-1, and GSDMD were measured to evaluate the protective mechanism of MT on ROSdependent pyroptosis in LPS-treated H9C2 cells. LPS treatment increased the expression of NLRP3, caspase-1, and GSDMD compared with the control group, and this effect was reversed by $\mathrm{MT}$ pretreatment (Figure $7 A, B, C, D$ ).

\section{Discussion}

In this study, MT pretreatment had shown a beneficial effect on LPS-induced myocardial injury. MT significantly improved cell viability by inhibiting inflammation and reducing the levels of IL- 6 , IL- $1 \beta$, TNF- $\alpha$, and ROSdependent cell pyroptosis. Moreover, MT inhibited the activation of the NF- $\mathrm{KB}$ pathway and reduced the expression of inflammatory proteins, such as iNOS and COX-2, and downregulated the expression of pyroptosis proteins including NLRP3, caspase-1, and GSDMD in myocardiocytes. These findings suggest that the protective effects of MT might be related to the inhibition of NF$\kappa \mathrm{B} / \mathrm{iNOS} / \mathrm{COX}-2$-dependent inflammatory responses and ROS-dependent pyroptosis.

The inflammatory response plays a vital role in myocardial injury (31). Cardiac dysfunction is closely related to inflammatory cytokines including IL-6 and TNF- $\alpha$ in sepsis $(32,33)$. Clinical and experimental studies have indicated that inflammatory cytokines trigger sepsisinduced cardiac dysfunction $(34,35)$, and inhibition of inflammatory cytokines (IL-6, TNF- $\alpha$ ) can improve cardiac dysfunction in the septic animal model and in patients with sepsis $(36,37)$. LPS-induced inflammatory responses could also be alleviated by MT via decreasing the release of inflammatory cytokines (38). In the present study, the experimental data indicated that MT reduced the production of the inflammatory cytokines (TNF- $\alpha$, 

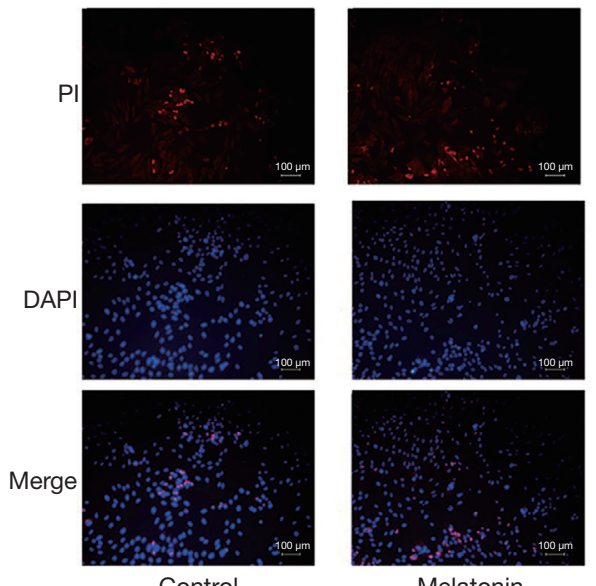

Melatonin
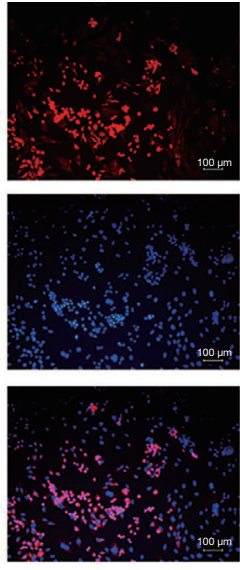

LPS
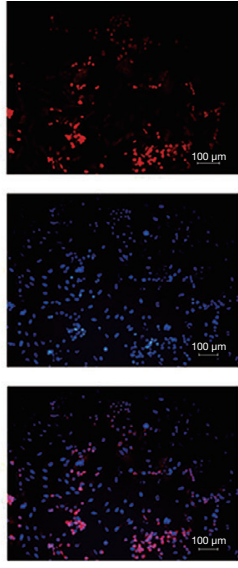

Melatonin + LPS

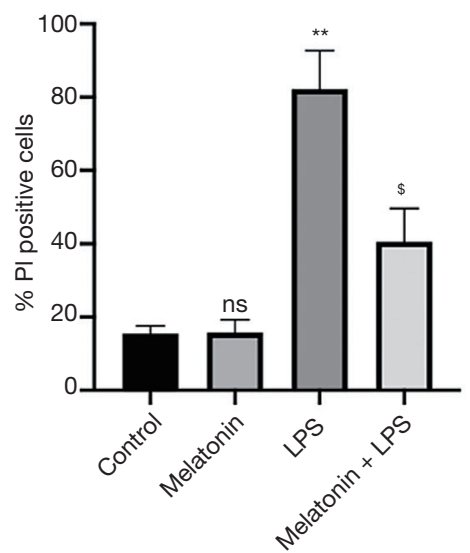

Figure 6 The effect of melatonin on pyroptotic cell death in H9C2 cells after lipopolysaccharide (LPS) treatment. Pyroptotic cells were detected using propidium iodide (PI) staining (scale bar: $100 \mu \mathrm{m} .100 \times$ magnification). Data are shown as mean \pm standard deviation (SD) $(\mathrm{n}=6) .{ }^{* *}, \mathrm{P}<0.01$ compared to control group; ${ }^{\$}, \mathrm{P}<0.05$ compared to LPS group; $\mathrm{ns}, \mathrm{P}>0.05$ compared to control group.

A

NLRP3

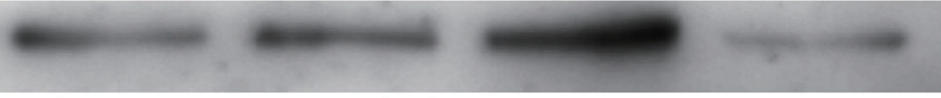

Caspase-1

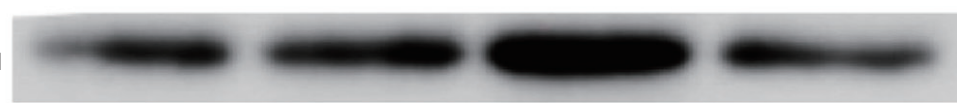

GSDMD

\section{GAPDH}

\section{LPS} Melatonin
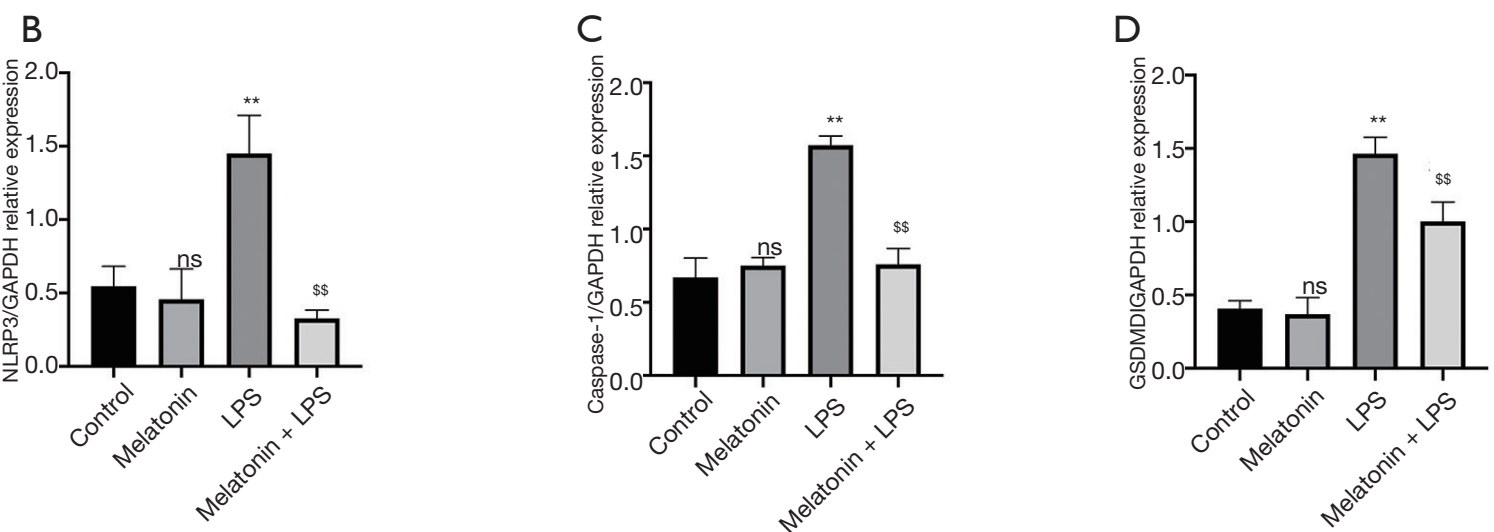

Figure 7 The effect of melatonin on the expression of NLR family pyrin domain containing 3 (NLRP3), caspase-1, and Gasdermin-D (GSDMD) in lipopolysaccharide (LPS)-treated H9C2 cells. (A) NLRP3, Caspase-1 and GSDMD protein expression in H9C2 was analyzed by western blot. The relative protein expression levels of (B) NLRP3/GAPDH, (C) caspase-1/GAPDH and (D) GSDMD/GAPDH in H9C2 cells were analyzed. Data are shown as mean \pm standard deviation $(\mathrm{SD})(\mathrm{n}=3)$. ${ }^{* *}, \mathrm{P}<0.01$ compared to control group; ${ }^{\mathrm{s}}, \mathrm{P}<0.01$ compared to LPS group; ns, P>0.05 compared to control group. 
IL-6), and improved the viability of $\mathrm{H} 9 \mathrm{C} 2$ cells by decreasing LDH.

MT showed a strong anti-inflammatory effect, thus, its effect on inflammation in H9C2 cells after LPS stimulation was further investigated. Inflammation and inflammatory cytokine release is closely related to COX-2 and

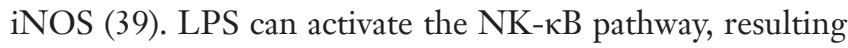
in inflammation (40). Furthermore, phosphorylation

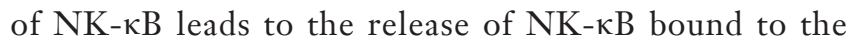
inhibitor $I \kappa B(41)$ and the translocation of dimerized $\mathrm{NK}-\kappa \mathrm{B}$ to the nucleus, thus promoting the expression of inflammatory genes including inflammatory cytokines (42). This is an important process which modulates inflammatory responses and drives the expression of iNOS and COX-2 (43). Previous studies have suggested that the expression of iNOS and COX-2 was mainly regulated by NF- $\kappa \mathrm{B}$ pathway, which was closely associated with myocardial dysfunction (44). In addition, MT could inhibit the expression of COX-2 and iNOS $(45,46)$, which also alleviates tissue injury by inhibiting the NF- $\mathrm{KB}$ pathway (24) with significantly decreased inflammation responses. However, the anti-inflammation mechanism of MT in LPSinduced myocardial injury is unclear. Our experimental results revealed that the upregulation of iNOS, COX2 , and $\mathrm{p}-\mathrm{NF}-\mathrm{\kappa B}$ could be effectively inhibited by MT in LPS-induced myocardial injury. Therefore, the protective effect of MT might be associated with its anti-inflammatory effects.

Oxidative stress is another important mechanism that mediates cardiovascular diseases, including myocardial injury (47). Recent studies have suggested that excessive ROS generation leads to LPS-induced cardiomyocyte injury $(48,49)$. Excessive generation of ROS and MDA and decreased activity of antioxidant enzymes such as SOD have been observed during oxidative stress (50). This can cause damage to cellular components and macromolecules, including proteins and DNA, and can induce mitochondrial dysfunction, ultimately leading to tissue damage (51). Our results suggested that MT could improve LPS-induced cell injury by decreasing the generation of ROS and MDA, and increasing the activity of the antioxidant enzyme SOD.

The NLRP3 inflammasome could be activated by ROSdependent signaling pathways after stimulation by PAMPs such as LPS (19). ROS are regarded as vital messengers that promote inflammasome activation. They have also been recognized to play an essential role in NLRP3 inflammasome activation (52) and drive NLRP3/caspase-1 complex activation, leading to pyroptosis (53). Under physiological conditions, ROS are eliminated by antioxidant enzymes during cell metabolism, maintaining the balance between ROS generation and elimination (54). When ROS are continuously generated, the intracellular antioxidant defense systems cannot maintain redox homeostasis, which eventually leads to oxidative stress-induced injury and cellular damage, including cell apoptosis (55) and vascular endothelial cell pyroptosis $(21,56)$. Our results suggested that the high-level production of ROS triggered cardiomyocyte pyroptosis, along with increased expression of NLRP3, caspase-1, and GSDMD. On the other hand, the data indicated that MT pretreatment reduced the generation of ROS, thus leading to the decreased expression of NLRP3, caspase-1, and GSDMD proteins, and reduced the release of IL-1 $\beta$. Therefore, the protective effect of MT might be associated with its antioxidant properties, which reduces the generation of ROS and, in turn, alleviates NLRP3-mediated pyroptosis in H9C2 cardiomyocytes. However, the protective mechanisms of MT in LPSinduced myocardial injury should be further elucidated in septic animal model, which is a limitation inherent to the research.

\section{Conclusions}

The present study demonstrated that MT pretreatment alleviated LPS-induced myocardial injury. Its protective effect might be related to its strong ability to inhibit NF$\kappa \mathrm{B} / \mathrm{iNOS} / \mathrm{COX}-2$-induced inflammation and ROSdependent pyroptosis in $\mathrm{H} 9 \mathrm{C} 2$ cardiomyocytes.

\section{Acknowledgments}

Funding: This study was supported by grants from National Natural Science Foundation of China (grant No. 82002014), Medical Science and Technology Research Funding of Guangdong (grant No. A2019409), the Fundamental Research Funds for the Central Universities (grant No. 2019MS136), Science and Technology Projects of Guangzhou (grant No. 201704020124, 201903010097) and Guangdong Provincial Key Laboratory of Coronary Heart Disease Prevention (grant No. 2017B030314041). The funders had no role in the study design, data collection and analysis, decision to publish, nor preparation of the manuscript.

\section{Footnote}

Reporting Checklist: The authors have completed the MDAR 


\section{Page 10 of 12}

checklist. Available at http://dx.doi.org/10.21037/atm-208196

Data Sharing Statement: Available at http://dx.doi. org/10.21037/atm-20-8196

Conflicts of Interest: All authors have completed the ICMJE uniform disclosure form (available at http://dx.doi. org/10.21037/atm-20-8196). The authors have no conflicts of interest to declare.

Ethical Statement: The authors are accountable for all aspects of the work in ensuring that questions related to the accuracy or integrity of any part of the work are appropriately investigated and resolved.

Open Access Statement: This is an Open Access article distributed in accordance with the Creative Commons Attribution-NonCommercial-NoDerivs 4.0 International License (CC BY-NC-ND 4.0), which permits the noncommercial replication and distribution of the article with the strict proviso that no changes or edits are made and the original work is properly cited (including links to both the formal publication through the relevant DOI and the license). See: https://creativecommons.org/licenses/by-nc-nd/4.0/.

\section{References}

1. Cecconi M, Evans L, Levy M, et al. Sepsis and septic shock. Lancet 2018;392:75-87.

2. Charpentier J, Luyt CE, Fulla Y, et al. Brain natriuretic peptide: A marker of myocardial dysfunction and prognosis during severe sepsis. Critical care medicine 2004;32:660-5.

3. Prescott HC, Angus DC. Enhancing Recovery From Sepsis: A Review. JAMA 2018;319:62-75.

4. Ehrman RR, Sullivan AN, Favot MJ, et al. Pathophysiology, echocardiographic evaluation, biomarker findings, and prognostic implications of septic cardiomyopathy: a review of the literature. Critical Care 2018;22:112.

5. Frencken JF, Donker DW, Spitoni C, et al. Myocardial Injury in Patients With Sepsis and Its Association With Long-Term Outcome. Circ Cardiovasc Qual Outcomes 2018;11:e004040.

6. Turdi S, Han X, Huff AF, et al. Cardiac-specific overexpression of catalase attenuates lipopolysaccharideinduced myocardial contractile dysfunction: role of autophagy. Free Radic Biol Med 2012;53:1327-38.
Su et al. The role of melatonin in LPS-induced myocardial injury

7. Jiang $\mathrm{W}, \mathrm{Luo} \mathrm{F}, \mathrm{Lu} \mathrm{Q}$, et al. The protective effect of Trillin LPS-induced acute lung injury by the regulations of inflammation and oxidative state. Chem Biol Interact 2016;243:127-34.

8. Merx MW, Weber C. Sepsis and the heart. Circulation 2007;116:793-802.

9. Matsuno K, Iwata K, Matsumoto M, et al. NOX1/NADPH oxidase is involved in endotoxin-induced cardiomyocyte apoptosis. Free Radic Biol Med 2012;53:1718-28.

10. Ward PA. The sepsis seesaw: seeking a heart salve. Nat Med 2009;15:497-8.

11. Kang R, Zeng L, Zhu S, et al. Lipid Peroxidation Drives Gasdermin D-Mediated Pyroptosis in Lethal Polymicrobial Sepsis. Cell Host Microbe 2018;24:97108.e4.

12. Qiu Z, He Y, Ming H, et al. Lipopolysaccharide (LPS) Aggravates High Glucose- and Hypoxia/ReoxygenationInduced Injury through Activating ROS-Dependent NLRP3 Inflammasome-Mediated Pyroptosis in H9C2 Cardiomyocytes. J Diabetes Res 2019;2019:8151836.

13. Liu X, Zhang Z, Ruan J, et al. Inflammasome-activated gasdermin $\mathrm{D}$ causes pyroptosis by forming membrane pores. Nature 2016;535:153-8.

14. He Y, Hara H, Nunez G. Mechanism and Regulation of NLRP3 Inflammasome Activation. Trends Biochem Sci 2016;41:1012-21.

15. Horng T. Calcium signaling and mitochondrial destabilization in the triggering of the NLRP3 inflammasome. Trends Immunol 2014;35:253-61.

16. Jia $\mathrm{C}$, Chen $\mathrm{H}$, Zhang J, et al. Role of pyroptosis in cardiovascular diseases. Int Immunopharmacol 2019;67:311-8.

17. Jin Y, Li H, Xie G, et al. Sevoflurane combined with ATP activates caspase- 1 and triggers caspase-1-dependent pyroptosis in murine $\mathrm{J} 774$ macrophages. Inflammation 2013;36:330-6.

18. Shi J, Zhao Y, Wang K, et al. Cleavage of GSDMD by inflammatory caspases determines pyroptotic cell death. Nature 2015;526:660-5.

19. Song W, Wei L, Du Y, et al. Protective effect of ginsenoside metabolite compound $\mathrm{K}$ against diabetic nephropathy by inhibiting NLRP3 inflammasome activation and NF-kappaB/p38 signaling pathway in high-fat diet/streptozotocin-induced diabetic mice. Int Immunopharmacol 2018;63:227-38.

20. Hardeland R, Pandi-Perumal SR, Cardinali DP. Melatonin. Int J Biochem Cell Biol 2006;38:313-6.

21. Arendt J, Bojkowski C, Folkard S, et al. Some effects of 
melatonin and the control of its secretion in humans. Ciba Found Symp. 1985;117:266-83.

22. Yang Y, Sun Y, Yi W, et al. A review of melatonin as a suitable antioxidant against myocardial ischemiareperfusion injury and clinical heart diseases. J Pineal Res 2014;57:357-66.

23. Hung MW, Kravtsov GM, Lau CF, et al. Melatonin ameliorates endothelial dysfunction, vascular inflammation, and systemic hypertension in rats with chronic intermittent hypoxia. J Pineal Res 2013;55:247-56.

24. Liu Z, Gan L, Xu Y, et al. Melatonin alleviates inflammasome-induced pyroptosis through inhibiting NF$\kappa \mathrm{B} / \mathrm{GSDMD}$ signal in mice adipose tissue. J Pineal Res 2017. doi: 10.1111/jpi.12414.

25. Zhang Y, Liu X, Bai X, et al. Melatonin prevents endothelial cell pyroptosis via regulation of long noncoding RNA MEG3/miR-223/NLRP3 axis. J Pineal Res 2018. doi: 10.1111/jpi.12449.

26. Pan P, Zhang H, Su L, et al. Melatonin Balance the Autophagy and Apoptosis by Regulating UCP2 in the LPS-Induced Cardiomyopathy. Molecules 2018;23:675.

27. Zhai M, Li B, Duan W, et al. Melatonin ameliorates myocardial ischemia reperfusion injury through SIRT3dependent regulation of oxidative stress and apoptosis. J Pineal Res 2017. doi: 10.1111/jpi.12419.

28. Zhang Y, Wang Y, Xu J, et al. Melatonin attenuates myocardial ischemia-reperfusion injury via improving mitochondrial fusion/mitophagy and activating the AMPK-OPA1 signaling pathways. J Pineal Res 2019;66:e12542.

29. Di S, Wang Z, Hu W, et al. The Protective Effects of Melatonin Against LPS-Induced Septic Myocardial Injury: A Potential Role of AMPK-Mediated Autophagy. Front Endocrinol (Lausanne) 2020;11:162.

30. Zhong J, Tan Y, Lu J, et al. Therapeutic contribution of melatonin to the treatment of septic cardiomyopathy: A novel mechanism linking Ripk3-modified mitochondrial performance and endoplasmic reticulum function. Redox Biol 2019;26:101287.

31. Frangogiannis NG. The inflammatory response in myocardial injury, repair, and remodelling. Nat Rev Cardiol 2014;11:255-65.

32. Zhang T, Feng Q. Nitric oxide and calcium signaling regulate myocardial tumor necrosis factor-alpha expression and cardiac function in sepsis. Can J Physiol Pharmacol 2010;88:92-104.

33. Geoghegan-Morphet N, Burger D, Lu X, et al. Role of neuronal nitric oxide synthase in lipopolysaccharide- induced tumor necrosis factor-alpha expression in neonatal mouse cardiomyocytes. Cardiovasc Res 2007;75:408-16.

34. Kumar V, Sharma A. Is neuroimmunomodulation a future therapeutic approach for sepsis? Int Immunopharmacol 2010;10:9-17.

35. Liu CJ, Lo JF, Kuo CH, et al. Akt mediates 17betaestradiol and/or estrogen receptor-alpha inhibition of LPS-induced tumor necresis factor-alpha expression and myocardial cell apoptosis by suppressing the JNK1/2NFkappaB pathway. J Cell Mol Med 2009;13:3655-67.

36. Vincent JL, Bakker J, Marecaux G, et al. Administration of anti-TNF antibody improves left ventricular function in septic shock patients. Results of a pilot study. Chest 1992;101:810-5.

37. Herbertson MJ, Werner HA, Goddard CM, et al. Antitumor necrosis factor-alpha prevents decreased ventricular contractility in endotoxemic pigs. Am J Respir Crit Care Med 1995;152:480-8.

38. Nabavi SM, Nabavi SF, Sureda A, et al. Anti-inflammatory effects of Melatonin: A mechanistic review. Crit Rev Food Sci Nutr 2019;59:S4-S16.

39. You R, Long W, Lai Z, et al. Discovery of a potential antiinflammatory agent: 3-oxo-29-noroleana-1,9(11),12-trien2,20-dicarbonitrile. J Med Chem 2013;56:1984-95.

40. Kang SR, Han DY, Park KI, et al. Suppressive Effect on Lipopolysaccharide-Induced Proinflammatory Mediators by Citrus aurantium L. in Macrophage RAW 264.7 Cells via NF-kappaB Signal Pathway. Evid Based Complement Alternat Med 2011;2011:248592.

41. de Martin R, Vanhove B, Cheng Q, et al. Cytokineinducible expression in endothelial cells of an I kappa B alpha-like gene is regulated by NF kappa B. EMBO J 1993;12:2773-9.

42. Kregel KC, Zhang HJ. An integrated view of oxidative stress in aging: basic mechanisms, functional effects, and pathological considerations. Am J Physiol Regul Integr Comp Physiol 2007;292:R18-36.

43. Lou T, Jiang W, Xu D, et al. Inhibitory Effects of Polydatin on Lipopolysaccharide-Stimulated RAW 264.7 Cells. Inflammation 2015;38:1213-20.

44. Yang P, Han Y, Gui L, et al. Gastrodin attenuation of the inflammatory response in $\mathrm{H} 9 \mathrm{c} 2$ cardiomyocytes involves inhibition of NF-kappaB and MAPKs activation via the phosphatidylinositol 3-kinase signaling. Biochem Pharmacol 2013;85:1124-33.

45. Yao C, Liu X, Zhou Z, et al. Melatonin attenuates expression of cyclooxygenase-2 (COX-2) in activated microglia induced by lipopolysaccharide (LPS). J Toxicol 
Page 12 of 12

Environ Health A 2019;82:437-46.

46. Hsu JT, Le PH, Lin CJ, et al. Mechanism of salutary effects of melatonin-mediated liver protection after trauma-hemorrhage: p38 MAPK-dependent iNOS/HIF$1 \alpha$ pathway. Am J Physiol Gastrointest Liver Physiol 2017;312:G427-G433.

47. Zhang Y, Murugesan P, Huang K, et al. NADPH oxidases and oxidase crosstalk in cardiovascular diseases: novel therapeutic targets. Nat Rev Cardiol 2020;17:170-94.

48. Bao J, Ye C, Zheng Z, et al. Fmr1 protects cardiomyocytes against lipopolysaccharide-induced myocardial injury. Exp Ther Med 2018;16:1825-33.

49. Gölz L, Memmert S, Rath-Deschner B, et al. LPS from $\mathrm{P}$. gingivalis and hypoxia increases oxidative stress in periodontal ligament fibroblasts and contributes to periodontitis. Mediators Inflamm 2014;2014:986264.

50. Lowes DA, Webster NR, Murphy MP, et al. Antioxidants that protect mitochondria reduce interleukin-6 and oxidative stress, improve mitochondrial function, and reduce biochemical markers of organ dysfunction in a rat model of acute sepsis. Br J Anaesth 2013;110:472-80.

51. Bian Q, Gao S, Zhou J, et al. Lutein and zeaxanthin supplementation reduces photooxidative damage and modulates the expression of inflammation-related genes in retinal pigment epithelial cells. Free Radic Biol Med

Cite this article as: $\mathrm{Su} Z \mathrm{ZDZ}$, Wei $\mathrm{XB}, \mathrm{Fu} \mathrm{YB}, \mathrm{Xu} \mathrm{J}$, Wang ZH, Wang Y, Cao JF, Huang JL, Yu DQ. Melatonin alleviates lipopolysaccharide-induced myocardial injury by inhibiting inflammation and pyroptosis in cardiomyocytes. Ann Transl Med 2021;9(5):413. doi: 10.21037/atm-20-8196

\section{Su et al. The role of melatonin in LPS-induced myocardial injury}

2012;53:1298-307.

52. Yu X, Lan P, Hou X, et al. HBV inhibits LPS-induced NLRP3 inflammasome activation and IL-1beta production via suppressing the NF-kappaB pathway and ROS production. J Hepatol 2017;66:693-702.

53. Muñoz-Planillo R, Kuffa P, Martinez-Colon G, et al. K(+) efflux is the common trigger of NLRP3 inflammasome activation by bacterial toxins and particulate matter. Immunity 2013;38:1142-53.

54. Wang X, Lai R, Su X, et al. Edaravone attenuates lipopolysaccharide-induced acute respiratory distress syndrome associated early pulmonary fibrosis via amelioration of oxidative stress and transforming growth factor-beta1/Smad3 signaling. Biochem Biophys Res Commun 2018;495:706-12.

55. Ding ZM, Jiao XF, Wu D, et al. Bisphenol AF negatively affects oocyte maturation of mouse in vitro through increasing oxidative stress and DNA damage. Chem Biol Interact 2017;278:222-9.

56. Wu X, Zhang H, Qi W, et al. Nicotine promotes atherosclerosis via ROS-NLRP3-mediated endothelial cell pyroptosis. Cell Death Dis 2018;9:171.

(English Language Editor: C. Betlazar-Maseh) 